\title{
A FAKE TOPOLOGICAL HILBERT SPACE \\ BY
}

R. D. ANDERSON, D. W. CURTIS AND J. VAN MILL \begin{abstract}
following properties:
(i) $X$ imbeds as a convex subset of $l^{2}$;

(ii) every compact subset of $X$ is a $Z$-set;

(iii) $X \times X \approx l^{2}$;

(iv) $X$ is homogeneous;

(v) $X \approx X \backslash G$ for every countable subset $G$.
\end{abstract}

ABSTRACT. We give an example of a topologically complete separable metric AR space $X$ which is not homeomorphic to the Hilbert space $l^{2}$, but which has the

1. Introduction. Torunczyk [15] has recently obtained the following topological characterization of the separable Hilbert space $l^{2}$.

1.1. TheOREM. A topologically complete separable metric AR space $X$ is homeomorphic to $l^{2}$ if and only if every map $f: \bigoplus_{1}^{\infty} Q_{i} \rightarrow X$ of the countable free union of Hilbert cubes into $X$ is strongly approximable by maps $g: \bigoplus_{1}^{\infty} Q_{i} \rightarrow X$ for which the collection $\left\{g\left(Q_{i}\right)\right\}$ is discrete.

This extremely useful characterization has now become the standard method for recognizing topological Hilbert spaces, in situations ranging from hyperspaces to infinite products to topological groups (see [7, 15, 9]). The above approximation property, referred to as the strong discrete approximation property, can be stated in various equivalent ways:

1.2. For each map $f: \bigoplus_{1}^{\infty} Q_{i} \rightarrow X$ and each open cover $\mathcal{Q}$ of $X$, there exists a map $g: \bigoplus_{1}^{\infty} Q_{i} \rightarrow X$ such that $f$ and $g$ are $\mathscr{Q}$-close and $\left\{g\left(Q_{i}\right)\right\}$ is discrete.

1.3. With respect to some admissible metric $d$ on $X$, for each map $f: \oplus_{1}^{\infty} Q_{i} \rightarrow X$ and each map $\varepsilon: X \rightarrow(0, \infty)$, there exists a map $g: \bigoplus_{1}^{\infty} Q_{i} \rightarrow X$ such that $d(f(y), g(y))<\varepsilon(f(y))$ for each $y$ and $\left\{g\left(Q_{i}\right)\right\}$ is discrete.

1.4. With respect to every admissible metric $d$ on $X$, for each map $f: \bigoplus_{1}^{\infty} Q_{i} \rightarrow X$ and each $\varepsilon>0$, there exists a map $g: \bigoplus_{1}^{\infty} Q_{i} \rightarrow X$ such that $d(f(y), g(y))<\varepsilon$ for each $y$ and $\left\{g\left(Q_{i}\right)\right\}$ is discrete.

The equivalence of (1.2) through (1.4) is well known and easily demonstrated (to show that $(1.4) \Rightarrow(1.2)$ use Theorem 9.4 in $[10$, p. 196]).

The example we describe in $\$ \S 3-6$ shows that the strong discrete approximation property cannot be relaxed by considering only positive constants $\varepsilon>0$ and a fixed

Received June 12, 1980 and, in revised form, April 10, 1981.

1980 Mathematics Subject Classification. Primary 54G20; Secondary 54C55, 54F65.

Key words and phrases. Hilbert space, absolute retract, discrete approximation property, Z-set, homogeneity, negligible subset. 
metric $d$ on $X$. Specifically, our example is a topologically complete separable metric AR space $(X, d)$ with the following properties.

(1) For each map $f: \oplus_{1}^{\infty} Q_{i} \rightarrow X$ and $\varepsilon>0$, there exists a map $g$ : $\bigoplus_{1}^{\infty} Q_{i} \rightarrow X$ such that $d(f(q), g(q))<\varepsilon$ for each $q$ and $\left\{g\left(Q_{i}\right)\right\}$ is discrete (we call this the weak discrete approximation property for $(X, d))$;

(2) every compact subset of $X$ is a $Z$-set (which implies that $X$ is nowhere locally-compact);

(3) $X$ imbeds as a convex subset of $l^{2}$;

(4) $X \times X \approx l^{2}$;

(5) $X$ is strongly locally homogeneous (which implies that $X$ is homogeneous and countable dense homogeneous);

(6) every countable subset of $X$ is strongly negligible (in particular, $X \backslash$ \{countable set $\} \approx X)$

(7) no Cantor set is negligible in $X$.

Since in $l^{2}$ every compact set is negligible [12], property (7) shows that $X \approx l^{2}$. Thus $X$ is a counterexample for the problems $T C 3$ and $A N R 5$ of [11]. Liem [14] has previously shown that the condition $X \times X \approx l^{2}$ does not imply $X \approx l^{2}$. His example, however, is not homogeneous (there exists $p \in X$ such that $\{p\}$ is not a $Z$-set, while $X \backslash\{p\}$ is an $l^{2}$-manifold).

In $\$ 7$ we state another criterion by which our example may be seen to be nonhomeomorphic to $l^{2}$, and which leads to the related construction of a counterexample to the Capset Characterization Theorem of [13].

2. Definition and terminology. Let $Q=\Pi_{1}^{\infty}[-1,1]_{i}$ and $s=\Pi_{1}^{\infty}(-1,1)_{i}$. The space $s$ is homeomorphic to $l^{2}[1]$, and is called the pseudo-interior of $Q$. We sometimes write $I_{i}$ for $[-1,1]_{i}$. On these product spaces we use the standard metric $d\left(\left(x_{i}\right),\left(y_{i}\right)\right)=\Sigma_{1}^{\infty} 2^{-i}\left|x_{i}-y_{i}\right|$.

Let $\mathcal{Q}$ be an open cover of a space $X$. Maps $f, g: Y \rightarrow X$ are थ-close if, for each $y \in Y$, there exists $U \in \mathcal{Q}$ containing both $f(y)$ and $g(y)$. For a metric $d$ on $X$, we write $d(f, g)<\varepsilon$ if $d(f(y), g(y))<\varepsilon$ for each $y \in Y$. A map $h: X \rightarrow X$ is limited by

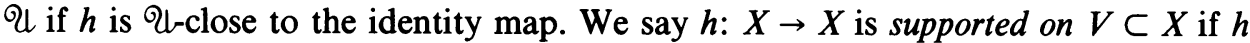
restricts to the identity map on $X \backslash V$.

A collection $\mathscr{D}$ of closed subsets of $X$ is discrete if each point of $X$ has a neighborhood intersecting at most one member of $\mathscr{D}$.

A closed subset $A \subset X$ is a $Z$-set in $X$ if, for each map $f: Q \rightarrow X$ and $\varepsilon>0$, there exists a map $g: Q \rightarrow X$ with $d(f, g)<\varepsilon$ and $g(Q) \cap A=\varnothing$. In $l^{2}$ every compact set is a $Z$-set. A $\sigma$-Z-set in $X$ is a countable union of $Z$-sets. We will use the fact, easily shown, that for every $\sigma-Z$-set $B \subset Q$, there exists a deformation $\alpha: Q \times[0,1] \rightarrow Q$ with $\alpha(q, 0)=q$ and $\alpha(q, t) \in Q \backslash B$, for all $q \in Q$ and $t>0$.

A space $X$ is strongly locally homogeneous if it has an open base $U$ such that, for each $U \in \mathcal{U}$ and points $x, y \in U$, there exists a homeomorphism $h: X \rightarrow X$ with $h(x)=y$ and $h$ supported on $U$. Clearly, every connected strongly locally homogeneous space is homogeneous.

A subset $K \subset X$ is negligible in $X$ if $X \approx X \backslash K . K$ is strongly negligible if there exist homeomorphisms $h: X \rightarrow X \backslash K$ limited by arbitrary open covers of $X$. In $l^{2}$, a subset $K$ is strongly negligible if and only if $K$ is a $\sigma$-Z-set [3]. 
3. Complements of $\sigma$ - $Z$-sets in $Q$. For each $i=1,2, \ldots$, let

$$
W_{i}=\prod_{j \neq i}\left[-1+2^{-i}, 1-2^{-i}\right]_{j} \times\{1\}_{i} \subset Q
$$

$W_{i}$ is a "shrunken endface" in the $i$ th coordinate direction, and is a $Z$-set in $Q$. We will show that the space $Y=Q \backslash \cup_{1}^{\infty} W_{i}$ has the properties specified in $\S 1$, except for the negligibility property (6). It then follows that, for any countable dense set $D \subset Y$, the space $X=Y \backslash D$ has all the required properties (Theorem 5.3). The results of this section will be used in verifying the properties (1)-(4), and (7).

3.1. TheOREM. Let $B \subset Q$ be a o-Z-set. Then $Q \backslash B$ is an infinite-dimensional topologically complete separable metric AR which imbeds as a convex subset of $l^{2}$.

Proof. We consider the "elliptic" Hilbert cube $K=\left\{\left(x_{i}\right) \in l^{2}: \Sigma_{1}^{\infty} i^{2} x_{i}^{2} \leqslant 1\right\}$. There exists a homeomorphism $h: Q \rightarrow K$ such that $h(B) \subset\left\{\left(x_{i}\right) \in l^{2}: \Sigma_{1}^{\infty} i^{2} x_{i}^{2}=1\right\}$ [5]. It is easily seen that $K \backslash h(B)$ is convex. It follows by Dugundji's Extension Theorem that $Q \backslash B \approx K \backslash h(B)$ is an AR. (An alternate proof that $Q \backslash B$ is an AR follows from the existence of a deformation $\alpha: Q \times[0,1] \rightarrow Q$ with $\alpha(Q \times(0,1]) \subset$ $Q \backslash B$.) $Q \backslash B$ is topologically complete since $B$ is $\sigma$-compact. The converse of this theorem is also true [9].

3.2. THEOREM. Let $B \subset Q$ be a $\sigma$-Z-set such that for each $\varepsilon>0$ there exists a map $\Re: Q \rightarrow B$ with $d(\Re$, id $)<\varepsilon$. Then the metric space $(Q \backslash B, d)$ has the weak discrete approximation property.

Proof. Let a map $f: \oplus_{1}^{\infty} Q_{i} \rightarrow Q \backslash B$ and $\varepsilon>0$ be given. Choose $\Re: Q \rightarrow B$ such that $d(\mathscr{N}$, id $)<\varepsilon$. Since $B$ is a $\sigma-Z$-set, there exist maps of $Q$ into $Q \backslash B$ arbitrarily close to the identity map. Composing the map $\Re$ with such maps, we obtain a sequence of maps $\left\{g_{i}: Q \rightarrow Q \backslash B\right\}$ such that

(i) $d\left(g_{i}, \Re\right)<1 / i$;

(ii) $d\left(g_{i}\right.$,id) $<\varepsilon$;

(iii) $g_{i}(Q) \cap g_{j}(Q)=\varnothing$ if $i \neq j$.

Define $g$ : $\oplus_{1}^{\infty} Q_{i} \rightarrow Q \backslash B$ by $g(q)=g_{i}(f(q))$, for $q \in Q_{i}$. Then $d(f, g)<\varepsilon$, and the collection $\left\{g\left(Q_{i}\right)\right\}=\left\{g_{i}\left(f\left(Q_{i}\right)\right)\right\}$ is discrete in $Q \backslash B$.

3.3. TheOREM. Let $(X, d)$ be a metric space with the weak discrete approximation property. Then every compact subset of $X$ is a Z-set.

Proof. Consider a compact subset $K$, and let a map $f: Q \rightarrow X$ and $\varepsilon>0$ be given. Define $\hat{f}: \bigoplus_{1}^{\infty} Q_{i} \rightarrow X$ by $\hat{f}(q)=f(q)$. By hypothesis there exists a map $g: \bigoplus_{1}^{\infty} Q_{i} \rightarrow X$ with $d(\hat{f}, g)<\varepsilon$ and $\left\{g\left(Q_{i}\right)\right\}$ a discrete collection. Since $K$ is compact, $K \cap g\left(Q_{i}\right)=$ $\varnothing$ for almost all $i$. Thus $K$ is a $Z$-set.

A $\sigma$-Z-set $B \subset Q$ for which $Q \backslash B \approx s \approx l^{2}$ is called a boundary set. In [8], various necessary and sufficient conditions are given for a $\sigma$ - $Z$-set to be a boundary set. Here, we use the following result.

3.4. LeMma. Let $B \subset Q$ be a $\sigma$-Z-set for which there exists a deformation of $Q$ through $B$ (i.e., a deformation $\alpha: Q \times[0,1] \rightarrow Q$ with $\alpha(q, 0)=q$ and $\alpha(q, t) \in B$, for all $q \in Q$ and $t>0$ ). Then $B$ is a boundary set. 
3.5. TheOREM. Let $B \subset Q$ be a $\sigma$-Z-set such that for each $\varepsilon>0$ there exists a map $\Re: Q \rightarrow B$ with $d(\Re$, id $)<\varepsilon$. Then $(Q \backslash B) \times(Q \backslash B) \approx l^{2}$.

Proof. We construct a deformation $\left\{\alpha_{t}\right\}$ of $Q \times Q$ through the $\sigma$-Z-set $\tilde{B}=(B \times$ $Q) \cup(Q \times B)$. For each $i \geqslant 1$, choose a map $\eta_{i}: Q \rightarrow B$ with $d\left(\eta_{i}\right.$, id $)<1 / i$. Set $\alpha_{0}=\mathrm{id} \times \mathrm{id}, \alpha_{1 /(2 i-1)}=\eta_{i} \times \eta_{i}, \alpha_{1 / 2 i}=\eta_{i+1} \times \eta_{i}$, and $\alpha_{1 /(2 i+1)}=\eta_{i+1} \times \eta_{i+1}$. Define $\alpha_{t}$ for $1 /(2 i+1)<t<1 / 2 i$ or $1 / 2 i<t<1 /(2 i-1)$ by using the straightline homotopy in $Q$ between $\eta_{i}$ and $\eta_{i+1}$. It follows from 3.4 that $(Q \backslash B) \times(Q \backslash B)$ $=(Q \times Q) \backslash \tilde{B} \approx l^{2}$.

3.6. Lemma. Let $B_{1}$ and $B_{2}$ be $\sigma$-Z-sets in $Q$ such that $Q \backslash B_{1} \approx Q \backslash B_{2}$. Then there exist a compact space $M$ and monotone surjections $\Pi_{i}: M \rightarrow Q, i=1,2$, such that $\Pi_{1}^{-1}\left(B_{1}\right)=\Pi_{2}^{-1}\left(B_{2}\right)$.

Proof. Let $h: Q \backslash B_{1} \rightarrow Q \backslash B_{2}$ be a homeomorphism, and let $\Gamma \subset Q \times Q$ be the graph of $h$. Take $M=\bar{\Gamma}$, and $\Pi_{i}: M \rightarrow Q$ the projection maps, $i=1,2$. Clearly, $\Pi_{1}^{-1}\left(B_{1}\right)=\bar{\Gamma} \backslash \Gamma=\Pi_{2}^{-1}\left(B_{2}\right)$, and since $Q \backslash B_{i}$ is dense in $Q$, each $\Pi_{i}$ is onto. By symmetry, it suffices to verify that $\Pi_{1}$ is monotone. For $x \in Q \backslash B_{1}, \Pi_{1}^{-1}(x)$ is a point in $\Gamma$. Suppose that for some $x \in B_{1}, \Pi_{1}^{-1}(x)$ is not connected. Let $\Pi_{1}^{-1}(x)=$ $F \cup G$ be a separation, and choose disjoint open sets $U$ and $V$ in $M$ containing $F$ and $G$, respectively. Since $\Pi_{1}$ is a closed map, there exists an open neighborhood $W$ of $x$ in $Q$ such that $\Pi_{1}^{-1}(W) \subset U \cup V$, and we may assume $W$ is connected. Then $W \backslash B_{1}$ is also connected (in fact, path-connected: any path in $W$ between points of $W \backslash B_{1}$ may be deformed to a path in $W \backslash B_{1}$ via a deformation $\alpha: Q \times[0,1] \rightarrow Q$ such that $\left.\alpha(Q \times(0,1]) \subset Q \backslash B_{1}\right)$. But

$$
W \backslash B_{1}=\left(W \cap \Pi_{1}(U \cap \Gamma)\right) \cup\left(W \cap \Pi_{1}(V \cap \Gamma)\right)
$$

is a separation. Thus $\Pi_{1}$ and $\Pi_{2}$ are monotone.

4. Strong local homogeneity. Given points $p, q$ in $Y=Q \backslash \cup_{1}^{\infty} W_{i}$ we will construct a homeomorphism $h: Q \rightarrow Q$ such that $h(p)=q$ and $h\left(W_{i}\right)=W_{i}$ for each $i$. Then $h$ restricts to a homeomorphism of $Y$. Moreover, our constructions will show that $Y$ is strongly locally homogeneous.

4.1. THEOREM. Let $p$ and $q$ be points in s. Then there exists a homeomorphism $h$ : $Q \rightarrow Q$ such that $h(p)=q$ and $h\left(W_{i}\right)=W_{i}$ for each $i$.

Proof. We may assume $p_{i} \leqslant q_{i}$ for each $i$. Choose $a_{i}$ and $b_{i}$ such that

$$
-1<a_{i}<p_{i} \leqslant q_{i}<b_{i}<1 \text {. }
$$

Let $H_{i}: I_{i} \times[0,1] \rightarrow I_{i}$ be an isotopy such that

(i) each level $H_{i, t}$ is supported on $\left[a_{i}, b_{i}\right]$;

(ii) $H_{i, 0}=$ id;

(iii) $H_{i, 1}\left(p_{i}\right)=q_{i}$.

For each $i$, let $B_{i}=\prod_{1}^{i} I_{j}$ and $D_{i}=\prod_{1}^{i}\left[a_{j}, b_{j}\right]$. There exists a map $\alpha_{i}: B_{i} \rightarrow[0,1]$ such that

(a) $\alpha_{i}\left(D_{i}\right)=1$;

(b) $\alpha_{i}\left(\partial B_{i}\right)=0$; 
(c) if $\left(s_{j}\right),\left(t_{j}\right) \in B_{i}$ such that for each $j$ either $s_{j}=t_{j}$ or $\left\{s_{j}, t_{j}\right\} \subset\left[a_{j}, b_{j}\right]$, then $\alpha_{i}\left(\left(s_{j}\right)\right)=\alpha_{i}\left(\left(t_{j}\right)\right)$.

(Collapse each interval $\left[a_{j}, b_{j}\right]$ to a point, then apply a Urysohn map.)

For each $i$, choose an integer $\tilde{i} \geqslant i$ such that $\left[a_{i}, b_{i}\right] \subset\left[-1+2^{-\tilde{i}}, 1-2^{-\tilde{i}}\right]$. Let $\Pi_{i}: Q \rightarrow I_{i}$ be the projection map, with $\Pi_{i}(x)=x_{i}$. Define a homeomorphism $h_{i}$ : $Q \rightarrow Q$ by the formulas

(1) $\Pi_{j} h_{i}=\Pi_{j}$ if $j \neq i$

(2) $\Pi_{i} h_{i}(x)=H_{i}\left(x_{i}, \alpha_{i}\left(x_{1}, \ldots, x_{i}\right)\right)$.

The verification that $h_{i}$ is 1-1 uses conditions (i) and (c).

Finally, define $h: Q \rightarrow Q$ by $h=\lim _{i \rightarrow \infty}\left(h_{i} \circ \cdots \circ h_{1}\right)$. Clearly, $h$ is continuous and onto, and $h(p)=q$. Consider distinct points $x, y \in Q$. Suppose $x_{i} \neq y_{i}$, and suppose $h(x)$ and $h(y)$ agree in the first $i-1$ coordinates. Let

$$
\left(v_{1}, \ldots, v_{i-1}, x_{i}, \ldots, x_{i}\right) \text { and }\left(v_{1}, \ldots, v_{i-1}, y_{i}, \ldots, y_{i}\right)
$$

be the first $\tilde{i}$ coordinates of $\left(h_{i-1} \circ \cdots \circ h_{1}\right)(x)$ and $\left(h_{i-1} \circ \cdots \circ h_{1}\right)(y)$, respectively. If $\alpha_{\tilde{i}}\left(v_{1}, \ldots, x_{\tilde{i}}\right)=\alpha_{\tilde{i}}\left(v_{1}, \ldots, y_{\tilde{i}}\right)$, then $\Pi_{i} h(x) \neq \Pi_{i} h(y)$. And otherwise, by condition (c), we must have $x_{j} \neq y_{j}$ and $\left\{x_{j}, y_{j}\right\} \nsubseteq\left[a_{j}, b_{j}\right]$ for some $i \leqslant j \leqslant i$. Then by condition (i), $\Pi_{j} h(x) \neq \Pi_{j} h(y)$. Thus $h$ is $1-1$.

For $j \leqslant \tilde{i}, h_{i}$ restricts to the identity on $W_{j}$. For $j>\tilde{i}, h_{i}\left(W_{j}\right)=W_{j}$, since $\left[a_{i}, b_{i}\right] \subset\left[-1+2^{-j}, 1-2^{-j}\right]$. Thus $h\left(W_{j}\right)=W_{j}$ for each $j$.

4.2. REMARK. The above proof shows that if for some $n, U$ is a neighborhood of the product set $\prod_{1}^{n}\left[a_{j}, b_{j}\right] \times \prod_{n+1}^{\infty} I_{j}$, then the homeomorphism $h$ may be constructed so as to be supported on $U$.

To construct a homeomorphism of $Y$ sending a point $p \in Y \backslash s$ into $s$, we employ an inductive convergence procedure stated in [2]. For each homeomorphism $g$ of $Q$ and $\varepsilon>0$, let $\Re(g, \varepsilon)=\inf \{d(g(x), g(y)): d(x, y) \geqslant \varepsilon\}$. If $\left\{h_{i}\right\}$ is a sequence of homeomorphisms of $Q$ such that $d\left(h_{i}\right.$, id $)<3^{-i} \cdot \Re\left(h_{j} \circ \cdots \circ h_{i-1}, 2^{-j}\right)$ for each $j<i$, it is easily verified that $h=\lim _{i \rightarrow \infty}\left(h_{i} \circ \cdots \circ h_{1}\right)$ is a homeomorphism of $Q$.

4.3. ThEOREM. For every $p \in Y$ there exists a homeomorphism $h: Q \rightarrow Q$ such that $h(p) \in s$ and $h\left(W_{i}\right)=W_{i}$ for each $i$.

Note. The construction via the inductive convergence criterion of a homeomorphism of $Q$ sending an arbitrary point into the pseudo-interior $s$ is well known. We need only to carry out this standard construction with a little extra control to insure that each $W_{i}$ remains invariant.

Proof. Assume that homeomorphisms $h_{1}, \ldots, h_{i-1}$ of $Q$ have been defined such that

(a) $\Pi_{j} \circ h_{i-1} \circ \cdots \circ h_{1}(p) \in(-1,1)$ for each $j<i$;

(b) $h_{i-1} \circ \cdots \circ h_{1}\left(W_{j}\right)=W_{j}$ for each $j$.

For some sufficiently small $\delta>0$, we construct a homeomorphism $h_{i}$ such that

(1) $d\left(h_{i}\right.$, id $)<\delta$;

(2) $\Pi_{j} \circ h_{i}=\Pi_{j}$ for each $j<i$;

(3) $\Pi_{i} \circ h_{i} \circ \cdots \circ h_{1}(p) \in(-1,1)$;

(4) $h_{i}\left(W_{j}\right)=W_{j}$ for each $j$.

Then $h=\lim _{i \rightarrow \infty}\left(h_{i} \circ \cdots \circ h_{1}\right)$ is the desired homeomorphism. 
Let $y=h_{i-1} \circ \cdots \circ h_{1}(p)$. If $y_{i} \in(-1,1)$, take $h_{i}=$ id. Otherwise, assume $y_{i}=1$ (the case $y_{i}=-1$ is simpler). Since $y \notin W_{i}$, there exists an integer $k \neq i$ such that $y_{k} \notin\left[-1+2^{-i}, 1-2^{-i}\right]=\Pi_{k}\left(W_{i}\right)$. Suppose the inductive convergence procedure requires that $d\left(h_{i}, \mathrm{id}\right)<\delta$; choose $n>i, n \neq k$, such that $2^{-n}<\delta / 3$. The homeomorphism $h_{i}$ is constructed as the product of a homeomorphism $\tilde{h}_{i}$ on the 3-cube $I_{i} \times I_{n} \times I_{k}$ and the identity homeomorphism on the product of the remaining factors of $Q$. The homeomorphism $\tilde{h}_{i}$ will actually move only the $i$ th and the $n$th coordinates.

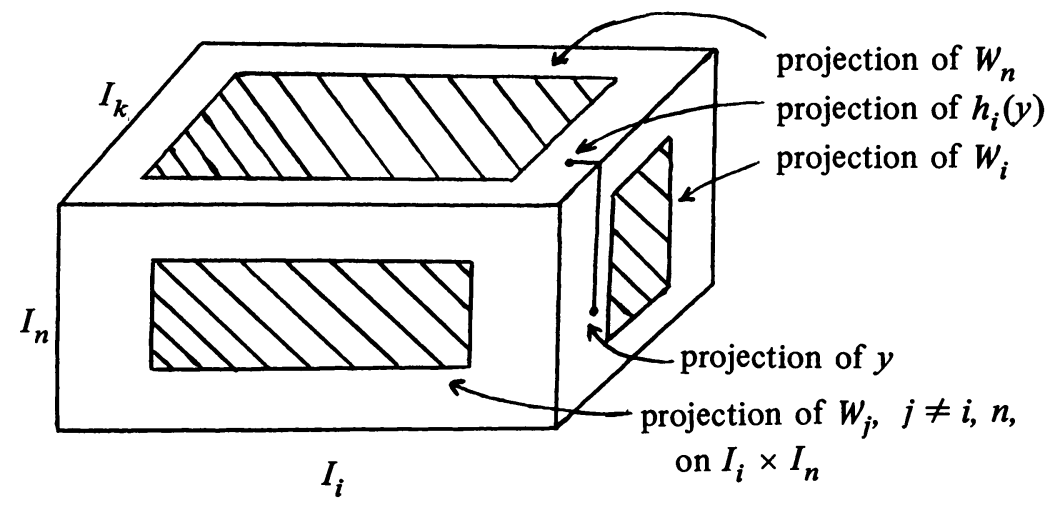

Let $D=\left[1-2^{-(n+1)}, 1\right] \times[-1,1] \subset I_{i} \times I_{n}$, and for each $0<r<1$ let $B_{r}=$ $[-1+r, 1-r] \times[-1+r, 1-r] \subset I_{i} \times I_{n}$. There exists an isotopy $H_{i}: I_{i} \times I_{n} \times$ $[0,1] \rightarrow I_{i} \times I_{n}$ with the following properties:

(i) $H_{i, 0}=\mathrm{id}$;

(ii) $H_{i, t}$ is supported on $D$ for all $t$;

(iii) $H_{i, t}\left(B_{r}\right)=B_{r}$ for all $r, t$;

(iv) $H_{i, 1}\left(\{1\} \times I_{n}\right) \subset\left(1-2^{-(n+1)}, 1\right) \times\{1\}$.

Let $\alpha: I_{k} \rightarrow[0,1]$ be a map such that $\alpha\left(\Pi_{k}\left(W_{i}\right)\right)=0$ and $\alpha\left(y_{k}\right)=1$. The homeomorphism $\tilde{h}_{i}$ on $I_{i} \times I_{n} \times I_{k}$ is then defined by $\tilde{h}_{i}\left(x_{i}, x_{n}, x_{k}\right)=$ $\left(H_{i}\left(x_{i}, x_{n}, \alpha\left(x_{k}\right)\right), x_{k}\right)$. The homeomorphism $h_{i}=\tilde{h}_{i} \times$ id on $Q$ has the specified properties (1)-(4). (In particular, $h_{i}$ restricts to the identity on $W_{i}$ and $W_{n}$, and takes each $W_{j}$ onto itself.)

4.4. REMARK. The above proof shows that the homeomorphism $h$ taking $p$ into $s$ may be constructed so as to be supported on a given neighborhood of $p$ (the role played by the map $\alpha: I_{k} \rightarrow[0,1]$ is taken over by a map $\left.\beta: I_{k_{1}} \times \cdots \times I_{k_{j}} \rightarrow[0,1]\right)$.

Combining 4.2 and 4.4, we conclude that $Y$ is strongly locally homogeneous.

5. Countable dense homogeneity. In this section we show that the strong local homogeneity of $Y$ implies that $Y$ is countable dense homogeneous, and therefore the space $X=Y \backslash$ \{countable dense subset\} has the property that $X \approx X \backslash$ \{countable subset . In fact, $X$ is also strongly locally homogeneous, and countable subsets of $X$ are strongly negligible.

A separable space $X$ is countably dense homogeneous if, for every pair $A, B$ of countable dense sets in $X$, there exists a homeomorphism of $X$ taking $A$ onto $B$. Bennett [4] showed that every countable dense homogeneous connected metric space 
is homogeneous, and that every strongly locally homogeneous locally compact separable metric space is countable dense homogeneous. The latter result is true more generally for topologically complete spaces. For its proof, we need a formulation of the inductive convergence procedure in a complete metric space.

5.1. LEMMA. Let $X$ be a complete metric space, and let $\left\{\mathscr{U}_{n}\right\}$ be a sequence of open covers and $\left\{h_{n}\right\}$ a sequence of homeomorphisms of $X$ satisfying the following conditions.

(1) $\mathcal{U}_{n}$ is a barycentric refinement of $\mathcal{U}_{n-1}$;

(2) $\mathcal{U}_{n}$ has mesh less than $2^{-n}$;

(3) $\left(h_{n-1} \circ \cdots \circ h_{1}\right)^{-1}\left(\mathscr{U}_{n}\right)$ has mesh less than $2^{-n}$;

(4) $h_{n}$ is limited by $\mathscr{Q}_{n}$.

Then $h=\lim _{n \rightarrow \infty}\left(h_{n} \circ \cdots \circ h_{1}\right)$ is a homeomorphism of $X$.

Note. A cover $\mathcal{V}$ of $X$ is a barycentric refinement of a cover $\mathscr{U}$ if $\{\operatorname{St}(x, \mathcal{V})$ : $x \in X\}$ refines $\mathcal{Q}$. Every open cover of a paracompact space has an open barycentric refinement.

Proof. Conditions (2) and (4) show that $h$ is a map with a dense image. We show that $h$ is $1-1$ and closed. Suppose that, for some $\varepsilon>0$, there exist sequences $\left\{x_{k}\right\}$ and $\left\{y_{k}\right\}$ in $X$ such that $d\left(x_{k}, y_{k}\right)>\varepsilon$ for each $k$ and $\lim _{k \rightarrow \infty} h\left(x_{k}\right)=z=\lim _{k \rightarrow \infty} h\left(y_{k}\right)$. Choose $n$ such that $2^{-n}<\varepsilon / 5$, and choose $U \in \mathcal{Q}_{n}$ such that $z \in U$. Then for some $k>n,\left\{h_{k} \circ \cdots \circ h_{1}\left(x_{k}\right), h_{k} \circ \cdots \circ h_{1}\left(y_{k}\right)\right\} \subset U$. By conditions (1) and (4), there exist $V, W \in \mathcal{Q}_{n}$ such that

$$
\left\{h_{n} \circ \cdots \circ h_{1}\left(x_{k}\right), h_{k} \circ \cdots \circ h_{1}\left(x_{k}\right)\right\} \subset V
$$

and

$$
\left\{h_{n} \circ \cdots \circ h_{1}\left(y_{k}\right), h_{k} \circ \cdots \circ h_{1}\left(y_{k}\right)\right\} \subset W .
$$

There also exist $V^{\prime}, W^{\prime} \in \mathscr{Q}_{n}$ such that

$$
\left\{h_{n-1} \circ \cdots \circ h_{1}\left(x_{k}\right), h_{n} \circ \cdots \circ h_{1}\left(x_{k}\right)\right\} \subset V^{\prime}
$$

and

$$
\left\{h_{n-1} \circ \cdots \circ h_{1}\left(y_{k}\right), h_{n} \circ \cdots \circ h_{1}\left(y_{k}\right)\right\} \subset W^{\prime} .
$$

Thus $\left(V^{\prime}, V, U, W, W^{\prime}\right)$ is a chain in $\mathscr{Q}_{n}$ with $h_{n-1} \circ \cdots \circ h_{1}\left(x_{k}\right) \in V^{\prime}$ and $h_{n-1} \circ \cdots \circ h_{1}\left(y_{k}\right) \in W^{\prime}$. Applying $\left(h_{n-1} \circ \cdots \circ h_{1}\right)^{-1}$, we obtain a 5-chain in $\left(h_{n-1} \circ \cdots \circ h_{1}\right)^{-1}\left(\mathscr{U}_{n}\right)$ between the points $x_{k}$ and $y_{k}$. By condition (3), $d\left(x_{k}, y_{k}\right)$ $<5 \cdot 2^{-n}<\varepsilon$, a contradiction. It follows that $h$ is $1-1$ and closed, and therefore a homeomorphism of $X$.

5.2. THEOREM. Every topologically complete separable metric space which is strongly locally homogeneous is countable dense homogeneous.

Proof. Let countable dense subsets $A=\left\{a_{1}, a_{2}, \ldots\right\}$ and $B=\left\{b_{1}, b_{2}, \ldots\right\}$ of such a space $X$ be given (assume $A$ and $B$ are faithfully indexed). The hypothesis of strong local homogeneity implies that for each neighborhood $U$ of a point $x$, and for any dense set $G$ in $X$, there exists a homeomorphism of $x$ which is supported on $U$ and takes $x$ into $G$. Using the inductive convergence procedure (with respect to some complete metric on $X$ ), we construct a sequence $\left\{h_{n}\right\}$ of homeomorphisms of $X$ such 
that $h=\lim _{n \rightarrow \infty}\left(h_{n} \circ \cdots \circ h_{1}\right)$ is a homeomorphism and such that the following conditions (which insure that $h(A)=B$ ) are satisfied.

(1) $h_{n} \circ \cdots \circ h_{1}\left(a_{i}\right)=h_{2 i} \circ \cdots \circ h_{1}\left(a_{i}\right) \in B$ for each $i$ and each $n \geqslant 2 i$;

(2) $\left(h_{n} \circ \cdots \circ h_{1}\right)^{-1}\left(b_{i}\right)=\left(h_{2 i+1} \circ \cdots \circ h_{1}\right)^{-1}\left(b_{i}\right) \in A$ for each $i$ and each $n \geqslant 2 i+1$.

Assume $h_{1}, \ldots, h_{2 i-1}$ have been defined. If $h_{2 i-1} \circ \ldots \circ h_{1}\left(a_{i}\right) \in B$, take $h_{2 i}=$ id. Otherwise, choose a neighborhood $U_{2 i}$ of $h_{2 i-1} \circ \cdots \circ h_{1}\left(a_{i}\right)$ disjoint from the finite set $\left\{b_{1}, \ldots, b_{i-1}\right\} \cup h_{2 i-1} \circ \ldots \circ h_{1}\left(\left\{a_{1}, \ldots, a_{i-1}\right\}\right)$, and take $h_{2 i}$ to be a homeomorphism of $X$ supported on $U_{2 i}$ and such that $h_{2 i} \circ \ldots \circ h_{1}\left(a_{i}\right) \in B$.

If $\left(h_{2 i} \circ \cdots \circ h_{1}\right)^{-1}\left(b_{i}\right) \in A$, take $h_{2 i+1}=$ id. Otherwise, choose a neighborhood $U_{2 i+1}$ of $b_{i}$ disjoint from the finite set $\left\{b_{1}, \ldots, b_{i-1}\right\} \cup h_{2 i} \circ \ldots \circ h_{1}\left(\left\{a_{1}, \ldots, a_{i}\right\}\right)$, and take $h_{2 i+1}$ to be a homeomorphism of $X$ supported on $U_{2 i+1}$ and such that $h_{2 i+1}^{-1}\left(b_{i}\right) \in\left(h_{2 i} \circ \cdots \circ h_{1}\right)^{-1}(A)$. If the neighborhoods $U_{2 i}$ and $U_{2 i+1}$ are chosen small enough, the conditions of the inductive convergence procedure are satisfied.

5.3. TheOREM. Let $Y$ be a topologically complete separable metric space which is strongly locally homogeneous, and let $D$ be a countable dense subset. Then $Y \backslash D$ is also strongly locally homogeneous, and every countable set in $Y \backslash D$ is strongly negligible.

Proof. Let $U$ be a neighborhood of a point $x$ in $Y \backslash D$. Then there exists an open neighborhood $V$ of $x$ in $Y$ with $V \backslash D \subset U$ and such that, for every $y \in V \backslash D$, there exists a homeomorphism $h_{y}$ of $Y$ supported on $V$ and taking $x$ to $y$. Then a homeomorphism $h=\lim _{n \rightarrow \infty}\left(h_{n} \circ \cdots \circ h_{1}\right)$ of $Y$ may be constructed as in the preceding lemma such that $h\left(h_{y}(D)\right)=D$, and each $h_{n}$ is supported on $V$ and leaves $y$ fixed. The restriction of $h \circ h_{y}$ to $Y \backslash D$ takes $x$ to $y$ and is supported on $V \backslash D$. Thus $Y \backslash D$ is strongly locally homogeneous.

Let $G \subset Y \backslash D$ be countable, and $\mathscr{U}$ an open cover of $Y \backslash D$. Then there is a countable collection $\mathcal{V}$ of pairwise disjoint open sets in $Y$ covering $G$, such that for each $V \in \mathfrak{V}, V \backslash D$ lies in a member of $\mathscr{Q}$. The proof of 5.2 shows that there exists a homeomorphism $h$ of $Y$ limited by the cover $\mathscr{V} \cup\{\{x\}: x \in Y\}$ and taking $D$ onto $D \cup G$. Then the restriction of $h$ to $Y \backslash D$ is a homeomorphism onto $(Y \backslash D) \backslash G$ which is limited by $\mathcal{Q}$.

Applying 5.3, we conclude that if $D$ is any countable dense subset of the space $Y=Q \backslash \cup_{1}^{\infty} W_{i}$, then the space $X=Y \backslash D$ has the homogeneity property (5) and the negligibility property (6) of $\S 1$. And by the theorems of $\S 3, X$ retains the properties (1) through (4). We show in the next section that $X$ has the nonnegligibility property (7).

\section{Nonnegligibility of Cantor sets.}

\subsection{TheOrem. No Cantor set is negligible in the space $X=Q \backslash\left(\cup_{1}^{\infty} W_{i} \cup D\right)$.}

Proof. Suppose $X \approx X \backslash K$ for some Cantor set $K$ in $X$. Then by Lemma 3.6 there exist a compact space $M$ and monotone surjections $\Pi_{1}: M \rightarrow Q$ and $\Pi_{2}: M \rightarrow Q$ such that $\Pi_{1}^{-1}\left(\cup_{1}^{\infty} W_{i} \cup D\right)=\Pi_{2}^{-1}\left(\cup_{1}^{\infty} W_{i} \cup D \cup K\right)$. For each $x \in K$, consider the continuum $\Pi_{2}^{-1}(x)$. Since $\Pi_{1}^{-1}\left(\cup W_{i} \cup D\right)$ is a countable disjoint union of compacta, and since no continuum is the countable infinite union of disjoint 
nonempty compacta (Sierpinski's Theorem), we must have $\Pi_{2}^{-1}(x) \subset \Pi_{1}^{-1}(C)$ for some $C \in\left\{W_{i}: i=1,2, \ldots\right\} \cup D$. Since $K$ is uncountable, there exist distinct points $x, y \in K$ such that $\Pi_{2}^{-1}(x) \cup \Pi_{2}^{-1}(y) \subset \Pi_{1}^{-1}(C)$ for some continuum $C$ as above. Since $\Pi_{1}^{-1}(C)$ is a continuum, $\Pi_{2}\left(\Pi_{1}^{-1}(C)\right) \subset Q$ is a continuum, and another application of Sierpinski's Theorem shows that $\Pi_{2}\left(\Pi_{1}^{-1}(C)\right) \subset K$. Thus $\Pi_{2}\left(\Pi_{1}^{-1}(C)\right)$ is a point, but $\{x, y\} \subset \Pi_{2}\left(\Pi_{1}^{-1}(C)\right)$, a contradiction.

Of course, the same argument applies as well to the space $Y=Q \backslash \cup_{1}^{\infty} W_{i}$. A similar argument shows that in $Y$, no countable infinite subset is negligible. In particular, $X=Y \backslash D \approx Y$.

\subsection{THEOREM. No countable infinite subset is negligible in the space $Y$.}

Proof. Suppose $Y \approx Y \backslash E$, where $E$ is countable infinite. There exist a compact space $M$ and monotone surjections $\Pi_{1}: M \rightarrow Q$ and $\Pi_{2}: M \rightarrow Q$ with $\Pi_{1}^{-1}\left(\cup_{1}^{\infty} W_{i}\right)$ $=\Pi_{2}^{-1}\left(\cup_{1}^{\infty} W_{i} \cup E\right)$. For each $x \in E, \Pi_{2}^{-1}(x) \subset \Pi_{1}^{-1}\left(W_{i}\right)$ for some $i$. Since $\Pi_{2}\left(\Pi_{1}^{-1}\left(W_{i}\right)\right)$ is a continuum, $\Pi_{2}\left(\Pi_{1}^{-1}\left(W_{i}\right)\right)=\{x\}$. Thus $\Pi_{2}^{-1}(x)=\Pi_{1}^{-1}\left(W_{i}\right)$. Consider the infinite subcollection $\mathscr{W}=\left\{W_{i}: \Pi_{1}^{-1}\left(W_{i}\right)=\Pi_{2}^{-1}(x)\right.$ for some $\left.x \in E\right\}$. Then $\cup$ is connected,

$$
\Pi_{1}^{-1}(\cup \mho)=\cup\left\{\Pi_{1}^{-1}\left(W_{i}\right): W_{i} \in \mathcal{W}\right\}=\bigcup\left\{\Pi_{2}^{-1}(x): x \in E\right\}=\Pi_{2}^{-1}(E)
$$

is connected, and $E=\Pi_{2}\left(\Pi_{2}^{-1}(E)\right)$ is connected, a contradiction.

7. A fake capset. As previously remarked, various characterizations of boundary sets (dense $\sigma$-Z-sets in $Q$ whose complements are homeomorphic to $s$ ) are given in [8]. One of these takes the following form.

\subsection{THEOREM. $A$ dense $\sigma-Z$-set $B \subset Q$ is a boundary set if and only if}

for every $\varepsilon>0$ there exists $\delta>0$ such that, for every compact set $K \subset B$ with $\operatorname{diam} K<\delta$, there exists a compact set $\tilde{K} \subset B$ with $\operatorname{diam} \tilde{K}<\varepsilon$ such that $K$ contracts to a point in every neighborhood of $\tilde{K}$ in $Q$.

Without loss of generality we may assume that $\tilde{K}$ is a continuum containing $K$. Thus, every boundary set is continuum-connected and locally continuum-connected.

This property of boundary sets provides another way of seeing that the spaces $Y=Q \backslash \cup_{1}^{\infty} W_{i}$ and $X=Q \backslash\left(\cup_{1}^{\infty} W_{i} \cup D\right)$ are not homeomorphic to $l_{2}$, since by Sierpinski's Theorem the $\sigma$-Z-sets $\cup_{1}^{\infty} W_{i}$ and $\cup_{1}^{\infty} W_{i} \cup D$ are not continuumconnected.

However, starting with the set $\cup_{1}^{\infty} W_{i}$, we may add a null sequence of arcs $\left\{\alpha_{i}\right\}$ in $Q$ to obtain a $\sigma$-Z-set $\cup_{1}^{\infty} W_{i} \cup\left(\cup_{1}^{\infty} \alpha_{i}\right)$ which is continuum-connected, but not locally continuum-connected. Taking products with copies of $Q$, we obtain the following.

7.2. EXAMPLE. There exists a tower of compacta $B_{1} \subset B_{2} \subset \ldots$ in $Q$ such that

(1) each $B_{n} \approx Q$;

(2) each $B_{n}$ is a $Z$-set in $Q$; 
(3) each $B_{n}$ is a $Z$-set in $B_{n+1}$;

(4) for every $\varepsilon>0$ there exists for some $n$ a map $\eta: Q \rightarrow B_{n}$ with $d(\eta$,id $)<\varepsilon$;

(5) $B=\cup_{1}^{\infty} B_{n}$ is not locally continuum-connected, and therefore $Q \backslash B \approx s$.

Note. The set $B$ is a counterexample to the Capset Characterization Lemma 1.1 of [13], which claimed that conditions (1) through (4) imply that $B$ is a capset (i.e., there exists a homeomorphism of $Q$ taking $B$ onto the pseudo-boundary $Q \backslash s$ ). The argument given there breaks down at the attempted application of the Anderson-Barit estimated homeomorphism extension theorem (every homeomorphism $h$ between $Z$-sets in $Q$ with $d(h$, id $)<\varepsilon$ can be extended to a homeomorphism $H$ of $Q$ with $d(H$, id $)<\varepsilon)$. Stated in this form, the extension theorem is valid only with respect to one of the standard convex metrics on $Q$. However, the application is attempted for the copies $B_{n}$ of $Q$, using the restrictions of a metric on $Q$, and these restrictions may be highly nonconvex.

The capset characterization theorem has been widely used by the authors, and others. Fortunately, in all applications of which we are aware, the mapping condition (4) can be replaced by the stronger condition

$\left(4^{*}\right)$ there exists a deformation $\alpha: Q \times[0,1] \rightarrow Q$ with $\alpha(q, 0)=q$ and such that, for every $t>0, \alpha(Q \times[t, 1]) \subset B_{n}$ for some $n$.

It is shown in [8] that if conditions (1) through (4*) are met, then $B=\cup_{1}^{\infty} B_{n}$ is a capset.

Construction of example. We first construct a tower $A_{1} \subset A_{2} \subset \ldots$ of compact AR's in $Q$ satisfying the conditions (4) and (5), and then take $B_{n}=A_{n} \times Q \times I^{n} \times$ $\{(1,1, \ldots)\} \subset Q \times Q \times Q$. By Edward's product theorem (see [6]), each $B_{n} \approx Q$.

For each $i=1,2, \ldots$, let

$$
\boldsymbol{\alpha}_{i}=\left(\{1\}_{i} \times\left[1-2^{-i}, 1\right]_{i+1} \cup\left[1-2^{-(i+1)}, 1\right]_{i} \times\{1\}_{i+1}\right) \times \prod_{j \neq i, i+1}\{0\}_{j} \subset Q .
$$

Then $\left\{\alpha_{\mathrm{i}}\right\}$ is a null sequence of disjoint arcs converging to the point $(0,0, \ldots)$; each $\alpha_{i}$ connects $W_{i}$ and $W_{i+1}$; and $\alpha_{i} \cap\left(\cup_{1}^{\infty} W_{j}\right)$ contains only the endpoints of $\alpha_{i}$. Take $A_{n}=\cup_{1}^{n}\left(W_{i} \cup \alpha_{i}\right)$. Clearly, $\cup_{1}^{\infty} A_{n}$ is not locally continuum-connected.

\section{REFERENCES}

1. R. D. Anderson, Hilbert space is homeomorphic to the countable infinite product of lines, Bull. Amer. Math. Soc. 72 (1966), 515-519.

2. __ On topological infinite deficiency, Michigan Math. J. 14 (1967), 365-383.

3. __ Strongly negligible sets in Fréchet manifolds, Bull. Amer. Math. Soc. 75 (1969), 64-67.

4. R. Bennett, Countable dense homogeneous spaces, Fund. Math. 74 (1972), 189-194.

5. C. Bessaga and A. Pelczyński, Selected topics in infinite-dimensional topology, PWN, Warsaw, 1975 .

6. T. A. Chapman, Lectures on Hilbert cube manifolds, CBMS Regional Conf. Series in Math., no. 28, Amer. Math. Soc., Providence, R. I., 1976.

7. D. W. Curtis, Hyperspaces homeomorphic to Hilbert space, Proc. Amer. Math. Soc. 75 (1979), $126-130$

8. Boundary sets in the Hilbert cube, in preparation.

9. T. Dobrowolski and H. Toruńczyk, Separable complete ANR's admitting a group structure are Hilbert manifolds, Topology Appl. 12 (1981), 229-235. 
10. J. Dugundji, Topology, Allyn and Bacon, Boston, Mass., 1966.

11. R. Geoghegan (editor), Open problems in infinite-dimensional topology, Topology Proc. 4 (1979), 287-338.

12. V. L. Klee, Convex bodies and periodic homeomorphisms in Hilbert space, Trans. Amer. Math. Soc. 74 (1953), 10-43.

13. N. Kroonenberg, Pseudo-interiors of hyperspaces, Compositio Math. 32 (1976), 113-131.

14. V. T. Liem, A counter-example in $l^{2}$-manifold theory, Proc. Amer. Math. Soc. 73 (1979), 119-120.

15. H. Torunczyk, Characterizing Hilbert space topology, Fund. Math. 111 (1981), 247-262.

Department of Mathematics, Louisiana State University, Baton Rouge, Louisiana 70803 